TRABAJO EN PROGRESO

\title{
Leetris: Un videojuego de apoyo al desarrollo de habilidades de comprensión de lectura
}

\author{
Manuel Soto Romero, Yoshua Ian Alfaro Mendoza, \\ David Alejandro Nieto Andrade
}

Publicado: 21 Septiembre 2016

\begin{abstract}
Resumen
El diseño de videojuegos tiene como principal objetivo crear una "experiencia" en el usuario que lo haga sentir interesado con las características del juego. Al diseñar videojuegos orientados a la educación se requieren satisfacer otras necesidades que permitan favorecer el aprendizaje esperado y que el jugador no pierda el interés en el mismo. En el presenta trabajo se muestra cómo, siguiendo el enfoque de la gamificación, se pueden diseñar videojuegos con objetivos didácticos, en particular para el desarrollo de habilidades de comprensión de lectura, de manera particular, presentamos el proceso de un videojuego llamado Leetris que intenta mejorar la comprensión de lectura mediante la resolución paralela de dos actividades cognitivas.
\end{abstract}

Palabras clave: Interacción Humano Computadora; Videojuegos; Gamificación; Experiencia del usuario.

\section{Introducción}

La lectura es una herramienta básica para el aprendizaje escolar y para seguir aprendiendo a lo largo de la vida [4]; de allí que, aprender a leer bien se ha convertido en una prioridad del ciudadano del siglo XXI. Para el caso de México y de acuerdo a los resultados de las evaluaciones realizadas por organismos internacionales (PISA, PEN Y Excale), el reto de formar lectores que comprenden lo que leen y lo usen en diferentes contextos dentro y fuera de la escuela, no se ha logrado. Considerando que la comprensión es la esencia de la lectura [4] se vuelve necesario encontrar estrategias innovadoras que promuevan el desarrollo de la comprensión de lectura.

Actualmente, se observa que los jóvenes están menos interesados en la lectura y prefieren estar frente a una pantalla explorando alguna de las nuevas experiencias que les proveen los videojuegos [1]. Por otra parte, una reciente encuesta realizada por el proyecto "Pew Internet \& American Life" [6] encontró que el $97 \%$ de los niños de entre 12 y 17 interactúan con juegos en diferentes dispositivos electrónicos como, consolas o smartphones. Basándonos en esta estadística consideramos que los

Soto Romero, M., Alfaro Mendoza, YI., Nieto Andrade, DA. Centro de Ciencias Aplicadas y Desarrollo Tecnológico, UNAM Circuito exterior S/N, C.P. 04510, Cd. Universitaria, Ciudad de México, Apdo. Postal 70-186

Email:manu@ciencias.unam.mx,yosh.alfaro@gmail.com,

davidalexito23@gmail.com videojuegos son una buena forma de acercarse a los adolescentes y promover su comprensión lectora.

Siguiendo estas ideas, este proyecto busca acercar a los jóvenes de bachillerato a la lectura a partir del uso de un videojuego que tiene el objetivo de mejorar sus habilidades de comprensión lectora.

\section{Uso de gamificación para fomentar interés en la lectura}

La gamificación consiste en aplicar mecánicas relacionadas con el juego a actividades no lúdicas con la finalidad de fomentar la motivación, la concentración, el esfuerzo y la fidelidad de un grupo de personas hacia la actividad de interés tales como estudio, realización de tareas, habilidades laborales, etc.

Existen videojuegos cuyo objetivo es fomentar el hábito de lectura mediante gamificación. Por ejemplo, el programa "Ranopla" dirigido a alumnos de primaria plantea leer libros y después responder un cuestionario asociado, se irán consiguiendo puntos de acuerdo al número de respuestas correctas [2].

En [10] se describe una metodología para diseñar una experiencia del usuario para aplicaciones orientadas al aprendizaje, siguiendo las fases propuestas desarrollaremos este proyecto.

\subsection{Resolución de dos problemas en paralelo}

La idea de resolver dos problemas en paralelo, pero no de forma simultánea, es atractiva para los usuarios, porque plantea un reto para sus habilidades. Actualmente encontramos este tipo propuestas en algunos juegos, por ejemplo, "Two cars game", el cual consiste en mover a dos carros por una pista, cada uno moviéndose en una mitad. De cada lado aparecen obstáculos que hay que evitar y objetos que deben de ser atrapados de manera asíncrona. El jugador debe estar concentrado en ambas tareas [3].

\subsection{Leetris: Justificación del diseño de la experiencia del usuario}

Considerando como base el objetivo de crear un desafío [5]; debemos de implementar una actividad que parezca difícil de resolver al principio, pero que se vuelve sencilla mientras más se adentre el usuario en el juego.

En Leetris, el desafío se centra, principalmente, en la comprensión de lectura; en un principio se presentan lecturas cortas de fácil comprensión y mientras se va avanzando entre los niveles se va aumentando la dificultad en el tipo de lectura. Por otro lado, tenemos un segundo desafío que es el jugar Tetris, al 
mismo tiempo que se contestan preguntas sobre la lectura. Para que nuestro desafío se considere divertido nos enfocamos en los siguientes puntos:

1. El usuario debe dividir su atención entre las dos tareas.

2. Buscar una experiencia envolvente a través de los elementos gráficos. la selección de lecturas y la presión para atender las dos tareas simultáneamente.

\section{Descripción del juego}

Leetris plantea resolver dos problemas: responder preguntas relacionadas a una lectura y jugar tetris en paralelo, de manera que las acciones en una de las tareas repercutan en la otra.

Está dirigido a jóvenes de entre 15 y 18 años de edad. El juego organiza las lecturas en cuatro mundos: romance, ciencia ficción, fantasía y terror, a su vez cada mundo tiene cuatro niveles que se deben recorrer en orden secuencial, ya que cada nivel presenta retos a las habilidades de lectura de una mayor dificultad, así el juego sigue generando preguntas y nuevos desafíos de Tetris para que vuelva el jugador [10].

Al elegir un nivel, se mostrará una lectura y cuando el jugador termina de leer indica que está listo. A continuación aparecerá la dinámica principal, que se compone de dos actividades: la actividad de la izquierda presenta la mecánica clásica del juego de tetris, mientras que la actividad de la derecha mostrará preguntas sobre la lectura de la pantalla anterior. Cada que el jugador conteste correctamente una pregunta, se dará un puntaje de 100 y se eliminará una línea. Por otro lado, si contesta mal, se disminuirá el tiempo en 5 segundos, se restará 100 puntos y se aumentará la velocidad de descenso de la figuras. Un juego que tenga un balance adecuado en los castigos hace que el juego sea más significativo y que los jugadores sientan orgullo cuando tengan éxito en el mismo [10].

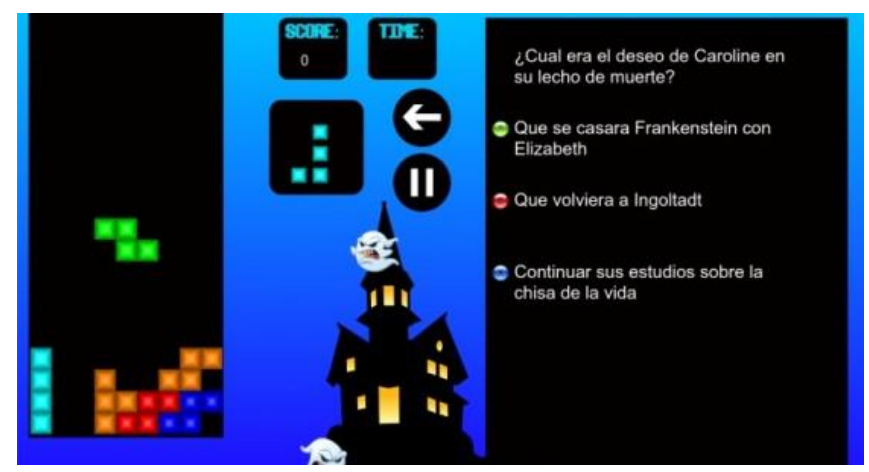

Figura 1. Pantalla con las dos actividades cognitivas

\section{Prueba del prototipo}

Se realizó una prueba de concepto con usuarios para ver si la mecánica propuesta en el juego era atractiva para los usuarios. La prueba sólo se centró en revisar lo atractivo de la experiencia en la propuesta, no se trató de medir una mejora en la comprensión lectora. Para esto se diseñó un prototipo digital usando un marco de trabajo para desarrollo de videojuegos llamado "Phaser"[9]. La prueba se realizó utilizando una cámara de Gesell en donde se pueden observar las acciones de los usuarios y en la cual jugaron la primera versión del prototipo.

En la evaluación participaron tres usuarios tipo, mujeres, en el rango de los dieciséis a los dieciocho años, mexicanas, estudiando el segundo o tercer año de bachillerato. Durante la sesión se les presentó el juego y se fue obteniendo su impresión de las diferentes pantallas. En la pantalla de la dinámica principal se les dio la oportunidad de jugar con el Tetris y resolver las preguntas sobre el texto de manera libre. Observamos que cada una de las usuarias jugó de manera diferente en esta sección: una de ellas enfocándose únicamente en las preguntas relacionadas al texto, la cual terminó rápidamente el nivel presentado y no mostró mucho interés en el juego; otra se enfocó en la sección del tetris y al descuidar la parte de las preguntas perdió; y por último, la tercera usuaria logró identificar las ventajas de trabajar en ambas tareas al mismo tiempo lo cual le generó mayor interés ante el desafío de resolver las dos tareas del juego.

Posteriormente a la prueba del juego se aplicaron cuestionarios con el objetivo de determinar si la experiencia que buscábamos transmitir era la correcta e identificar qué mejoras son necesarias. Las tres usuarias indicaron que las reglas no eran del todo claras, ya que no sabían que tenían que hacer las dos tareas de manera simultánea. Las tres estuvieron de acuerdo en el punto de que las mecánicas del juego lo hacían más divertido. Todas las usuarias contestaron de manera afirmativa a la pregunta que si recomendarían el juego a un amigo dándonos a entender que el juego fue de su agrado. Se observó que usar preguntas de opción múltiple, ayudó en general a los jugadores a reflexionar sobre la lectura al tratar de recordar la idea sobre la cual se les estaba preguntando.

\section{Observaciones y mejoras}

Con base en los resultados obtenidos en las pruebas y las expresiones de las tres usuarias, se está trabajando, principalmente en balancear la relación entre el Tetris y las preguntas. Para hacer esta relación más fuerte se redefinieron las reglas del juego tanto en los premios como en los castigos, del mismo modo se agregó tiempo para fortalecer la relación entre ambas tareas y finalmente la mejora en la interfaz de usuario en cuanto a los elementos gráficos.

Actualmente, se está trabajando en la implementación del resto de los niveles, se están seleccionando las lecturas adecuadas para los usuarios finales y que serán leídas desde archivos externos. Con estos cambios se planea hacer una prueba de la experiencia de los usuarios con una muestra más grande para obtener evidencia de su actitud hacia la lectura, antes y después del uso del videojuego.

\section{Agradecimientos}

Agradecemos los comentarios y el apoyo para la elaboración de este proyecto a Ana Libia Eslava Cervantes, Ricardo Castañeda Martínez, Jesús Ramírez Ortega y Gustavo de la Cruz Martínez del grupo Espacios y Sistemas Interactivos para la Educación del CCADET de la UNAM.

\section{Referencias}

[1] Boesner, Juliana. (2008). Los videojuegos y la promoción de la lectura. http://papelenblanco.com/. 30/junio/2016.

[2] De La Cruz, G., Eslava L., Castañeda R., (2015) Diseño de la Experiencia del Usuario para Espacios Interactivos de Aprendizaje no Formal.

[3] Delors J. (1998). La educación encierra un tesoro. Informe de la Comisión Internacional Sobre la Educación para el siglo XXI. http://goo.gl/MPDLJW. 9/junio/2016.

[4] Durkin D. (1993). Teaching them to read. Boston: Allyn and Baco

[5] Fullerton et al. (2008). Game Design Workshop: Designing, Protoyping and Playtesting Games. CMP Books, 2da edición.

[6] Ketchapp. "2 Cars". https://goo.gl/pWUABH. 28/junio/2016. 
[7] Lenhart, Amanda et al. (2008) Teens, Videogames and Civics. Pew Research Center: Internet Science \& Tech.

[8] López M. (2015). Programa Ranopla: Gamificación aplicada a la mejora de la comprensión lectora. http://goo.gl/O2QHsq 27/julio/2016.
[9] Richard Davey. "Phaser - A Fast, Fun and Free Open Source HTML5 Game Framework". http://phaser.io. 30/junio/2016.

[10] Schell Jesse. (2008). The Art Of Game Design. Morgan Kaufman 